\title{
Convergence results for the zero-finding problem and fixed points of nonexpansive semigroups and strict pseudocontractions
}

\section{Prasit Cholamjiak}

\section{"Correspondence:}

prasitch2008@yahoo.com

School of Science, University of

Phayao, Phayao, 56000, Thailand

\begin{abstract}
In this work, we establish strong convergence theorems for solving the fixed point problem of nonexpansive semigroups and strict pseudocontractions, and the zero-finding problem of maximal monotone operators in a Hilbert space. We further apply our result to the convex minimization problem and commutative semigroups.

MSC: $47 \mathrm{H} 09 ; 47 \mathrm{H} 10$
\end{abstract}

Keywords: fixed point; maximal monotone operator; left regular; strict pseudocontraction; nonexpansive semigroup

\section{Introduction}

Let $H$ be a real Hilbert space and $K$ a nonempty, closed, and convex subset of $H$. Let $T$ : $K \rightarrow K$ be a nonlinear mapping. Then $T$ is said to be nonexpansive if $\|T x-T y\| \leq\|x-y\|$ for all $x, y \in K$. The fixed points set of $T$ is denoted by $F(T)$.

In 1953, Mann [21] introduced the following classical iteration for a nonexpansive mapping $T: K \rightarrow K$ in a real Hilbert space: $x_{1} \in K$ and

$$
x_{n+1}=\alpha_{n} x_{n}+\left(1-\alpha_{n}\right) T x_{n}, \quad n \geq 1,
$$

where $\left\{\alpha_{n}\right\} \subset(0,1)$.

In 1967, Halpern [13] introduced another classical iteration for a nonexpansive mapping $T: K \rightarrow K$ in a real Hilbert space: $x_{1} \in K$ and

$$
x_{n+1}=\alpha_{n} u+\left(1-\alpha_{n}\right) T x_{n}, \quad n \geq 1,
$$

where $\left\{\alpha_{n}\right\} \subset(0,1)$ and $u \in K$ is fixed.

Let $f: K \rightarrow K$ be a contraction (i.e., $\|f(x)-f(y)\| \leq \alpha\|x-y\|$ for all $x, y \in K$ and $\alpha \in[0,1)$ ). In 2000, Moudafi [25] introduced the viscosity approximation method for a nonexpansive mapping $T$ as follows: $x_{1} \in K$ and

$$
x_{n+1}=\alpha_{n} f\left(x_{n}\right)+\left(1-\alpha_{n}\right) T x_{n}, \quad n \geq 1,
$$

where $\left\{\alpha_{n}\right\} \subset(0,1)$. It was proved, in a Hilbert space that the sequence $\left\{x_{n}\right\}$ generated by (1.2) strongly converges to a fixed point of $T$ under suitable conditions.

(c) 2012 Cholamjiak; licensee Springer. This is an Open Access article distributed under the terms of the Creative Commons Attribution License (http://creativecommons.org/licenses/by/2.0), which permits unrestricted use, distribution, and reproduction in any medium, provided the original work is properly cited. 
Let $A$ be a strongly positive bounded linear operator on $H$ : that is, there is a constant $\bar{\gamma}$ with property

$$
\langle A x, x\rangle \geq \bar{\gamma}\|x\|^{2} \quad \forall x \in H .
$$

A typical problem is to minimize a quadratic function over the set of the fixed points of a nonexpansive mapping on a real Hilbert space $H$ :

$$
\min _{x \in K}\langle A x, x\rangle-\langle x, b\rangle
$$

where $K$ is the fixed point set of a nonexpansive mapping $T$ on $H$ and $b$ is a given point in $H$.

Recently, Marino-Xu [22] introduced the following general iterative method for a nonexpansive mapping $T$ in a Hilbert space: $x_{1} \in H$ and

$$
x_{n+1}=\alpha_{n} \gamma f\left(x_{n}\right)+\left(I-\alpha_{n} A\right) T x_{n}, \quad n \geq 1,
$$

where $\left\{\alpha_{n}\right\} \subset(0,1), f$ is a contraction and $A$ is a strongly positive bounded linear operator.

Since then, there have been a number of modified viscosity approximation methods for nonexpansive mappings or nonexpansive semigroups (see, for example, $[6,7,9,26,32,35$, $38,42,43])$.

Recall that $T: K \rightarrow K$ is called a $\kappa$-strict pseudocontraction if there exists a constant $0 \leq \kappa<1$ such that

$$
\|T x-T y\|^{2} \leq\|x-y\|^{2}+\kappa\|(I-T) x-(I-T) y\|^{2}
$$

for all $x, y \in K$. It is known that (1.3) is equivalent to the following:

$$
\langle T x-T y, x-y\rangle \leq\|x-y\|^{2}-\frac{1-\kappa}{2}\|(I-T) x-(I-T) y\|^{2}
$$

for all $x, y \in K$.

The class of strict pseudocontractions was introduced, in 1967, by Browder-Petryshyn [3]. The existence and weak convergence theorems were proved in a real Hilbert space by using Mann iterative algorithm (1.1) with a constant sequence $\alpha_{n}=\alpha$ for all $n \geq 1$. Recently, Marino-Xu [23] and Zhou [44] extended the results of Browder-Petryshyn [3] to Mann's iteration process (1.1). Since 1967, the study of fixed points for strict pseudocontractions has been investigated by many authors (see, e.g., $[1,28]$ ).

A set-valued mapping $M: H \rightarrow 2^{H}$ is called monotone if for all $x, y \in H, f \in M(x)$, and $g \in M(y)$ imply $\langle x-y, f-g\rangle \geq 0$. A monotone mapping $M$ is maximal if its graph $G(M):=\{(f, x) \in H \times H: f \in M(x)\}$ of $M$ is not properly contained in the graph of any other monotone mapping. It is known that a monotone mapping $M$ is maximal if and only if for $(x, f) \in H \times H,\langle x-y, f-g\rangle \geq 0$ for all $(y, g) \in G(M)$ imply $f \in M(x)$. Let $J_{\lambda}^{M}=(I+\lambda M)^{-1}$, $\lambda>0$ be the resolvent of $M$. It is well known that $J_{\lambda}^{M}$ is single-valued and $D\left(J_{\lambda}^{M}\right)=H$ for any $\lambda>0$. For each $\lambda>0$, the Yosida approximation of $M$ is defined by $A_{\lambda}=\frac{I-J_{\lambda}^{M}}{\lambda}$. We know that $\left(J_{\lambda}^{M} x, A_{\lambda} x\right) \in G(M)$ for all $\lambda>0$ and $x \in H$.

A fundamental problem of monotone operators is that of finding an element $x$ such that $0 \in M x$. Such a problem is called the zero-finding problem (denoted by $M^{-1}(0)$ the 
set of solutions) and also includes many concrete examples, such as convex programming and monotone variational inequalities. It is known that if $g: H \rightarrow(-\infty, \infty]$ is a proper lower semicontinuous convex function, then $\partial g$ is maximal monotone and the equation $0 \in \partial g(x)$ is reduced to $g(x)=\min \{g(y): y \in H\}$ (see $[29,30]$ ).

Initiated by Martinet [24], Rockafellar [30] introduced the following iterative scheme: $x_{1} \in H$ and

$$
x_{n+1}=J_{\lambda_{n}}^{M} x_{n}, \quad n \geq 1,
$$

where $\left\{\lambda_{n}\right\} \subset(0, \infty)$ and $M$ is a maximal monotone operator on $H$. Such an algorithm is called the proximal point algorithm. It was proved that the sequence $\left\{x_{n}\right\}$ generated by (1.4) converges weakly to an element in $M^{-1}(0)$ if $\liminf _{n \rightarrow \infty} \lambda_{n}>0$.

The convergence of the zero-finding problem of monotone operators has been studied by many authors in several setting (see, for example, [8, 10, 14, 15, 27, 34]).

In this work, motivated by Lau et al. [16-20], Marino-Xu [22], and Saeidi [32], we introduce a new general iterative scheme for solving the fixed-point problem of a nonexpansive semigroup involving a strict pseudocontraction and the zero-finding problem of a maximal monotone operator in the framework of a Hilbert space. Some applications concerning the convex minimization problem and commutative semigroups are also presented.

\section{Preliminaries and lemmas}

In this section, we state some preliminaries and lemmas which will be used in the sequel.

Let $S$ be a semigroup. We denote by $\ell^{\infty}(S)$ the Banach space of all bounded real-valued functionals on $S$ with supremum norm. For each $s \in S$, we define the left and right translation operators $l(s)$ and $r(s)$ on $\ell^{\infty}(S)$ by

$$
(l(s) f)(t)=f(s t) \quad \text { and } \quad(r(s) f)(t)=f(t s)
$$

for each $t \in S$ and $f \in \ell^{\infty}(S)$, respectively. Let $X$ be a subspace of $\ell^{\infty}(S)$ containing 1 . An element $\mu$ in the dual space $X^{*}$ of $X$ is said to be a mean on $X$ if $\|\mu\|=\mu(1)=1$. It is well known that $\mu$ is a mean on $X$ if and only if

$$
\inf _{s \in S} f(s) \leq \mu(f) \leq \sup _{s \in S} f(s)
$$

for each $f \in X$. We often write $\mu_{t}(f(t))$ instead of $\mu(f)$ for $\mu \in X^{*}$ and $f \in X$.

Let $X$ be a translation invariant subspace of $\ell^{\infty}(S)$ (i.e., $l(s) X \subset X$ and $r(s) X \subset X$ for each $s \in S$ ) containing 1 . Then a mean $\mu$ on $X$ is said to be left invariant (resp. right invariant) if $\mu(l(s) f)=\mu(f)$ (resp. $\mu(r(s) f)=\mu(f)$ ) for each $s \in S$ and $f \in X$. A mean $\mu$ on $X$ is said to be invariant if $\mu$ is both left and right invariant [16-18]. $S$ is said to be left (resp. right) amenable if $X$ has a left (resp. right) invariant mean. $S$ is a amenable if $S$ is left and right amenable. In this case, $\ell^{\infty}(S)$ also has an invariant mean. It is known that $\ell^{\infty}(S)$ is amenable when $S$ is commutative semigroup or solvable group. However, the free group or semigroup of two generators is not left or right amenable (see $[11,20])$. A net $\left\{\mu_{\alpha}\right\}$ of means on $X$ is said to be left regular [11] if

$$
\lim _{\alpha}\left\|l_{s}^{*} \mu_{\alpha}-\mu_{\alpha}\right\|=0
$$

for each $s \in S$, where $l_{s}^{*}$ is the adjoint operator of $l_{s}$. 
Let $K$ be a nonempty, closed, and convex subset of $H$. A family $\mathcal{S}=\{T(s): s \in S\}$ is called a nonexpansive semigroup on $K$ if for each $s \in S$, the mapping $T(s): K \rightarrow K$ is nonexpansive and $T(s t)=T(s) T(t)$ for each $s, t \in S$. We denote by $F(\mathcal{S})$ the set of common fixed points of $\mathcal{S}$, i.e.,

$$
F(\mathcal{S})=\bigcap_{s \in S} F(T(s))=\bigcap_{s \in S}\{x \in K: T(s) x=x\} .
$$

Throughout this article, we denote the open ball of radius $r$ centered at 0 by $B_{r}$ and also denote the closed and convex hull of $A \subset H$ by $\overline{c o} A$. For $\varepsilon>0$ and a mapping $T: D \rightarrow H$, the set of $\varepsilon$-approximate fixed points of $T$ will be denoted by $F_{\varepsilon}(T, D)$, i.e. $F_{\varepsilon}(T, D)=\{x \in$ $D:\|x-T x\| \leq \varepsilon\}$.

The following lemmas are important in order to prove our main theorem.

Lemma 2.1 [20,31,39] Let $f$ be a function of a semigroup $S$ into a Banach space E such that the weak closure of $\{f(t): t \in S\}$ is weakly compact and let $X$ be a subspace of $\ell^{\infty}(S)$ containing all the functions $t \mapsto\left\langle f(t), x^{*}\right\rangle$ with $x^{*} \in E^{*}$. Then, for any $\mu \in X^{*}$, there exists a unique element $f_{\mu}$ in $E$ such that

$$
\left\langle f_{\mu}, x^{*}\right\rangle=\mu_{t}\left\langle f(t), x^{*}\right\rangle
$$

for all $x^{*} \in E^{*}$. Moreover, if $\mu$ is a mean on $X$ then

$$
\int f(t) d \mu(t) \in \overline{c o}\{f(t): t \in S\}
$$

We can write $f_{\mu}$ by $\int f(t) d \mu(t)$.

Lemma $2.2[20,31,39]$ Let $K$ be a closed and convex subset of a Hilbert space $H, \mathcal{S}=$ $\{T(s): s \in S\}$ be a nonexpansive semigroup from $K$ into $K$ such that $F(\mathcal{S}) \neq \emptyset$ and $X$ be a subspace of $\ell^{\infty}(S)$ containing 1 and the mapping $t \mapsto\langle T(t) x, y\rangle$ be an element of $X$ for each $x \in K$ and $y \in H$, and $\mu$ be a mean on $X$.

If we write $T(\mu) x$ instead of $\int T_{t} x d \mu(t)$, then the following hold:

(i) $T(\mu)$ is a nonexpansive mapping from $K$ into $K$;

(ii) $T(\mu) x=x$ for each $x \in F(\mathcal{S})$;

(iii) $T(\mu) x \in \overline{c o}\left\{T_{t} x: t \in S\right\}$ for each $x \in K$;

(iv) if $\mu$ is left invariant, then $T(\mu)$ is a nonexpansive retraction from $K$ onto $F(\mathcal{S})$.

Let $K$ be a nonempty, closed, and convex subset of a real Hilbert space $H$. Then, for any $x \in H$, there exists a unique nearest point in $K$, denoted by $P_{K} x$, such that

$$
\left\|x-P_{K} x\right\| \leq\|x-y\|
$$

for all $y \in K$. Such a projection $P_{K}$ is called the metric projection of $H$ onto $K$. We also know that for $x \in H$ and $z \in K, z=P_{K} x$ if and only if

$$
\langle x-z, y-z\rangle \leq 0, \quad \forall y \in K .
$$

We know the following subdifferential inequality. 
Lemma 2.3 For all $x, y \in H$, there holds the inequality

$$
\|x+y\|^{2} \leq\|x\|^{2}+2\langle y, x+y\rangle .
$$

Lemma 2.4 [22] Let $A$ be a strongly positive bounded linear operator on a Hilbert space $H$ with coefficient $\bar{\gamma}$ and $0<\rho \leq\|A\|^{-1}$. Then $\|I-\rho A\| \leq 1-\rho \bar{\gamma}$.

In the sequel, we need the following crucial lemmas.

Lemma 2.5 [41] Assume $\left\{a_{n}\right\}$ is a sequence of nonnegative real numbers such that

$$
a_{n+1} \leq\left(1-\rho_{n}\right) a_{n}+\rho_{n} \delta_{n}, \quad n \geq 1,
$$

where $\left\{\rho_{n}\right\}$ is a sequence in $(0,1)$ and $\left\{\delta_{n}\right\}$ is a sequence in $\mathbb{R}$ such that

(a) $\sum_{n=1}^{\infty} \rho_{n}=\infty$;

(b) $\lim \sup _{n \rightarrow \infty} \delta_{n} \leq 0$ or $\sum_{n=1}^{\infty}\left|\rho_{n} \delta_{n}\right|<\infty$.

Then $\lim _{n \rightarrow \infty} a_{n}=0$.

Lemma 2.6 [36] Let $\left\{x_{n}\right\}$ and $\left\{y_{n}\right\}$ be bounded sequences in a Banach space E such that

$$
x_{n+1}=\left(1-\beta_{n}\right) y_{n}+\beta_{n} x_{n}, \quad \forall n \geq 1,
$$

where $\left\{\beta_{n}\right\}$ is a real sequence in $(0,1)$ with $0<\liminf _{n \rightarrow \infty} \beta_{n} \leq \lim \sup _{n \rightarrow \infty} \beta_{n}<1$. If $\lim \sup _{n \rightarrow \infty}\left(\left\|y_{n+1}-y_{n}\right\|-\left\|x_{n+1}-x_{n}\right\|\right) \leq 0$, then $\lim _{n \rightarrow \infty}\left\|y_{n}-x_{n}\right\|=0$.

The following crucial results can be found in [1]

Lemma 2.7 [1] Let $K$ be a nonempty, closed, and convex subset of a real Hilbert space $H$ and let $T: K \rightarrow K$ be a $\kappa$-strict pseudocontraction such that $F(T) \neq \emptyset$, then $I-T$ is demiclosed at zero, that is, for all sequence $\left\{x_{n}\right\} \subset K$ with $x_{n} \rightarrow y$ and $\left\|x_{n}-T x_{n}\right\| \rightarrow 0$ it follows that $y=T y$.

Lemma 2.8 [1] Let $K$ be a nonempty, closed, and convex subset of a real Hilbert space $H$ and let $T_{i}: K \rightarrow K(i=1,2, \ldots, N)$ be a family of $\kappa_{i}$-strict pseudocontractions for some $0 \leq \kappa_{i}<1$. Assume $\left\{\eta_{i}\right\}_{i=1}^{N}$ is a positive sequence such that $\sum_{i=1}^{N} \eta_{i}=1$. Then $\sum_{i=1}^{N} \eta_{i} T_{i}$ is a $\kappa$-strict pseudocontraction with $\kappa=\max \left\{\kappa_{i}: 1 \leq i \leq N\right\}$. Moreover, if $\left\{T_{i}\right\}_{i=1}^{N}$ has a common fixed point, then $F\left(\sum_{i=1}^{N} \eta_{i} T_{i}\right)=\bigcap_{i=1}^{N} F\left(T_{i}\right)$.

Lemma 2.9 [40] Let the resolvent $J_{\lambda}^{M}$ be defined by $J_{\lambda}^{M}=(I+\lambda M)^{-1}, \lambda>0$. Then the following holds:

$$
\left\|J_{s}^{M} x-J_{t}^{M} x\right\| \leq\left|\frac{s-t}{t}\right|\left\|x-J_{t}^{M} x\right\|
$$

for all $s, t>0$ and $x \in H$. 


\section{Main result}

In this section, we are now ready to prove our main theorem.

Theorem 3.1 Let $H$ be a real Hilbert space and $\mathcal{S}=\{T(t): t \in S\}$ a nonexpansive semigroup on $H$. Let $M: H \rightarrow 2^{H}$ be a maximal monotone operator and $T: H \rightarrow H$ a $\kappa$-strict pseudocontraction such that $F:=F(\mathcal{S}) \cap M^{-1}(0) \cap F(T) \neq \emptyset$. Let $X$ be a left invariant subspace of $\ell^{\infty}(S)$ such that $1 \in X$, and the function $t \mapsto\langle T(t) x, y\rangle$ is an element of $X$ for each $x, y \in H$. Let $\left\{\mu_{n}\right\}$ be a left regular sequence of means on $X$ such that $\lim _{n \rightarrow \infty}\left\|\mu_{n+1}-\mu_{n}\right\|=0$. Let $f$ be an $\alpha$-contraction on $H$ and $A$ a strongly positive bounded linear operator with coefficient $\bar{\gamma}$. Let $\beta$ and $\gamma$ be real numbers such that $0<\beta<1$ and $0<\gamma<\bar{\gamma} / \alpha$. Let $\left\{x_{n}\right\}$ be generated by $x_{1} \in H$ and

$$
\left\{\begin{array}{l}
y_{n}=J_{\lambda_{n}}^{M}\left(\delta_{n} x_{n}+\left(1-\delta_{n}\right) T x_{n}\right), \\
x_{n+1}=\alpha_{n} \gamma f\left(x_{n}\right)+\beta x_{n}+\left((1-\beta) I-\alpha_{n} A\right) T\left(\mu_{n}\right) y_{n}, \quad n \geq 1,
\end{array}\right.
$$

where $\left\{\alpha_{n}\right\} \subset(0,1),\left\{\delta_{n}\right\} \subset(\kappa, 1)$ and $\left\{\lambda_{n}\right\} \subset(0, \infty)$ satisfying the conditions:

(C1) $\lim _{n \rightarrow \infty} \alpha_{n}=0$ and $\sum_{n=1}^{\infty} \alpha_{n}=\infty$;

(C2) $\lim _{n \rightarrow \infty}\left|\delta_{n+1}-\delta_{n}\right|=0$;

(C3) $\kappa<\liminf _{n \rightarrow \infty} \delta_{n} \leq \limsup _{n \rightarrow \infty} \delta_{n}<1$;

(C4) $\liminf _{n \rightarrow \infty} \lambda_{n}>0$ and $\lim _{n \rightarrow \infty}\left|\lambda_{n+1}-\lambda_{n}\right|=0$.

Then $\left\{x_{n}\right\}$ converges strongly to $p \in F$ which also solves the following variational inequality:

$$
\langle(\gamma f-A) p, q-p\rangle \leq 0, \quad \forall q \in F
$$

Proof Since $\alpha_{n} \rightarrow 0$, we shall assume that $\alpha_{n} \leq(1-\beta)\|A\|^{-1}$ and $1-\alpha_{n}(\bar{\gamma}-\alpha \gamma)>0$. So by Lemma 2.4, we have $\left\|(1-\beta) I-\alpha_{n} A\right\| \leq 1-\beta-\alpha_{n} \bar{\gamma}$.

First, we show that $\left\{x_{n}\right\}$ is bounded. Let $w \in F$. Put $z_{n}=\delta_{n} x_{n}+\left(1-\delta_{n}\right) T x_{n}$ for all $n \in \mathbb{N}$. Then

$$
\begin{aligned}
\left\|z_{n}-w\right\|^{2}= & \left\|\delta_{n} x_{n}+\left(1-\delta_{n}\right) T x_{n}-w\right\|^{2} \\
= & \left\|\delta_{n}\left(x_{n}-w\right)+\left(1-\delta_{n}\right)\left(T x_{n}-w\right)\right\|^{2} \\
= & \delta_{n}\left\|x_{n}-w\right\|^{2}+\left(1-\delta_{n}\right)\left\|T x_{n}-w\right\|^{2}-\delta_{n}\left(1-\delta_{n}\right)\left\|x_{n}-T x_{n}\right\|^{2} \\
\leq & \delta_{n}\left\|x_{n}-w\right\|^{2}+\left(1-\delta_{n}\right)\left\|x_{n}-w\right\|^{2}+\left(1-\delta_{n}\right) \kappa\left\|x_{n}-T x_{n}\right\|^{2} \\
& -\delta_{n}\left(1-\delta_{n}\right)\left\|x_{n}-T x_{n}\right\|^{2} \\
= & \left\|x_{n}-w\right\|^{2}+\left(1-\delta_{n}\right)\left(\kappa-\delta_{n}\right)\left\|x_{n}-T x_{n}\right\|^{2} \\
\leq & \left\|x_{n}-w\right\|^{2},
\end{aligned}
$$

which yields

$$
\left\|z_{n}-w\right\| \leq\left\|x_{n}-w\right\|
$$

Moreover, since $J_{\lambda_{n}}^{M}$ is firmly nonexpansive,

$$
\left\|y_{n}-w\right\|=\left\|J_{\lambda_{n}}^{M} z_{n}-w\right\| \leq\left\|z_{n}-w\right\| \leq\left\|x_{n}-w\right\| .
$$


From (3.3), we have

$$
\begin{aligned}
\left\|x_{n+1}-w\right\| \leq & \left\|\left[(1-\beta) I-\alpha_{n} A\right]\left[T\left(\mu_{n}\right) y_{n}-w\right]\right\|+\left\|\alpha_{n} \gamma\left[f\left(x_{n}\right)-f(w)\right]\right\| \\
& +\left\|\alpha_{n}[\gamma f(w)-A w]\right\|+\left\|\beta\left(x_{n}-w\right)\right\| \\
\leq & {\left[1-\alpha_{n}(\bar{\gamma}-\alpha \gamma)\right]\left\|x_{n}-w\right\|+\alpha_{n}\|\gamma f(w)-A w\| } \\
\leq & \max \left\{\left\|x_{n}-w\right\|, \frac{\|\gamma f(w)-A w\|}{(\bar{\gamma}-\gamma \alpha)}\right\} .
\end{aligned}
$$

By an induction, we can show that

$$
\left\|x_{n}-w\right\| \leq \max \left\{\left\|x_{1}-w\right\|, \frac{\|\gamma f(w)-A w\|}{(\bar{\gamma}-\gamma \alpha)}\right\}, \quad \forall n \geq 1
$$

Therefore, $\left\{x_{n}\right\}$ is bounded. So are $\left\{f\left(x_{n}\right)\right\},\left\{y_{n}\right\},\left\{z_{n}\right\}$, and $\left\{T\left(\mu_{n}\right) y_{n}\right\}$.

We next show that

$$
\lim _{n \rightarrow \infty}\left\|x_{n+1}-x_{n}\right\|=0
$$

Observe that

$$
\lim _{n \rightarrow \infty}\left\|T\left(\mu_{n+1}\right) y_{n}-T\left(\mu_{n}\right) y_{n}\right\|=0 \text {. }
$$

Indeed,

$$
\begin{aligned}
\left\|T\left(\mu_{n+1}\right) y_{n}-T\left(\mu_{n}\right) y_{n}\right\| & =\sup _{\|z\|=1}\left|\left\langle T\left(\mu_{n+1}\right) y_{n}-T\left(\mu_{n}\right) y_{n}, z\right\rangle\right| \\
& =\sup _{\|z\|=1}\left|\left(\mu_{n+1}\right)_{s}\left\langle T(s) y_{n}, z\right\rangle-\left(\mu_{n}\right)_{s}\left\langle T(s) y_{n}, z\right\rangle\right| \\
& \leq\left\|\mu_{n+1}-\mu_{n}\right\| \sup _{s \in S}\left\|T(s) y_{n}\right\| .
\end{aligned}
$$

Since $\left\{y_{n}\right\}$ is bounded and $\lim _{n \rightarrow \infty}\left\|\mu_{n+1}-\mu_{n}\right\|=0$, (3.4) holds.

For each $n \in \mathbb{N}$, define $T_{n} x=\delta_{n} x+\left(1-\delta_{n}\right) T x$. Then $T_{n}$ is nonexpansive, and hence

$$
\begin{aligned}
\left\|z_{n+1}-z_{n}\right\| & =\left\|T_{n+1} x_{n+1}-T_{n} x_{n}\right\| \\
& \leq\left\|T_{n+1} x_{n+1}-T_{n+1} x_{n}\right\|+\left\|T_{n+1} x_{n}-T_{n} x_{n}\right\| \\
& \leq\left\|x_{n+1}-x_{n}\right\|+\left|\delta_{n+1}-\delta_{n}\right| M_{1}
\end{aligned}
$$

for some big enough constant $M_{1}>0$.

On the other hand, since $y_{n}=J_{\lambda_{n}}^{M} z_{n}$ and $y_{n+1}=J_{\lambda_{n+1}}^{M} z_{n+1}$,

$$
\begin{aligned}
\left\|y_{n+1}-y_{n}\right\| & =\left\|J_{\lambda_{n+1}}^{M} z_{n+1}-J_{\lambda_{n}}^{M} z_{n}\right\| \\
& \leq\left\|J_{\lambda_{n+1}}^{M} z_{n+1}-J_{\lambda_{n}}^{M} z_{n+1}\right\|+\left\|J_{\lambda_{n}}^{M} z_{n+1}-J_{\lambda_{n}}^{M} z_{n}\right\| \\
& \leq\left\|J_{\lambda_{n+1}}^{M} z_{n+1}-J_{\lambda_{n}}^{M} z_{n+1}\right\|+\left\|z_{n+1}-z_{n}\right\| .
\end{aligned}
$$


Put $w_{n}=\frac{x_{n+1}-\beta x_{n}}{1-\beta}$. Then

$$
\begin{aligned}
w_{n+1}-w_{n}= & \frac{1}{1-\beta}\left[\left(x_{n+2}-\beta x_{n+1}\right)-\left(x_{n+1}-\beta x_{n}\right)\right] \\
= & \frac{1}{1-\beta}\left[\alpha_{n+1}\left(\gamma f\left(x_{n+1}\right)-A T\left(\mu_{n+1}\right) y_{n+1}\right)+(1-\beta) T\left(\mu_{n+1}\right) y_{n+1}\right] \\
& -\frac{1}{1-\beta}\left[\alpha_{n}\left(\gamma f\left(x_{n}\right)-A T\left(\mu_{n}\right) y_{n}\right)+(1-\beta) T\left(\mu_{n}\right) y_{n}\right] \\
= & \frac{\alpha_{n+1}}{1-\beta}\left(\gamma f\left(x_{n+1}\right)-A T\left(\mu_{n+1}\right) y_{n+1}\right)+T\left(\mu_{n+1}\right) y_{n+1} \\
& -\frac{\alpha_{n}}{1-\beta}\left(\gamma f\left(x_{n}\right)-A T\left(\mu_{n}\right) y_{n}\right)-T\left(\mu_{n}\right) y_{n} \\
= & \frac{\alpha_{n+1}}{1-\beta}\left(\gamma f\left(x_{n+1}\right)-A T\left(\mu_{n+1}\right) y_{n+1}\right)+\left(T\left(\mu_{n+1}\right) y_{n+1}-T\left(\mu_{n+1}\right) y_{n}\right) \\
& -\frac{\alpha_{n}}{1-\beta}\left(\gamma f\left(x_{n}\right)-A T\left(\mu_{n}\right) y_{n}\right)-\left(T\left(\mu_{n}\right) y_{n}-T\left(\mu_{n+1}\right) y_{n}\right)
\end{aligned}
$$

which implies

$$
\begin{aligned}
\left\|w_{n+1}-w_{n}\right\| \leq & \frac{\alpha_{n+1}}{1-\beta}\left\|\gamma f\left(x_{n+1}\right)-A T\left(\mu_{n+1}\right) y_{n+1}\right\|+\left\|y_{n+1}-y_{n}\right\| \\
& +\frac{\alpha_{n}}{1-\beta}\left\|\gamma f\left(x_{n}\right)-A T\left(\mu_{n}\right) y_{n}\right\|+\left\|T\left(\mu_{n}\right) y_{n}-T\left(\mu_{n+1}\right) y_{n}\right\| .
\end{aligned}
$$

Substituting (3.5) and (3.6) into (3.7), we obtain

$$
\begin{aligned}
\left\|w_{n+1}-w_{n}\right\| \leq & \frac{\alpha_{n+1}}{1-\beta}\left\|\gamma f\left(x_{n+1}\right)-A T\left(\mu_{n+1}\right) y_{n+1}\right\|+\left\|J_{\lambda_{n+1}}^{M} z_{n+1}-J_{\lambda_{n}}^{M} z_{n+1}\right\| \\
& +\left\|x_{n+1}-x_{n}\right\|+\left|\delta_{n+1}-\delta_{n}\right| M_{1}+\frac{\alpha_{n}}{1-\beta}\left\|\gamma f\left(x_{n}\right)-A T\left(\mu_{n}\right) y_{n}\right\| \\
& +\left\|T\left(\mu_{n}\right) y_{n}-T\left(\mu_{n+1}\right) y_{n}\right\| .
\end{aligned}
$$

Using Lemma 2.9, (3.4), (C1), (C2), and (C4), we have

$$
\limsup _{n \rightarrow \infty}\left(\left\|w_{n+1}-w_{n}\right\|-\left\|x_{n+1}-x_{n}\right\|\right) \leq 0
$$

From Lemma 2.6, we derive

$$
\lim _{n \rightarrow \infty}\left\|w_{n}-x_{n}\right\|=0
$$

It also follows that

$$
\lim _{n \rightarrow \infty}\left\|x_{n+1}-x_{n}\right\|=0
$$

We next show that

$$
\lim _{n \rightarrow \infty}\left\|x_{n}-T(t) x_{n}\right\|=0, \quad \forall t \in S .
$$


Put

$$
K=\max \left\{\left\|x_{1}-w\right\|, \frac{\|\gamma f(w)-A w\|}{(\bar{\gamma}-\gamma \alpha)}\right\} .
$$

Set $D=\{y \in H:\|y-w\| \leq K\}$. Then $D$ is a nonempty bounded closed convex set. Moreover, $\left\{x_{n}\right\},\left\{y_{n}\right\}$, and $\left\{z_{n}\right\}$ are in $D$. To complete our proof, we follow the proof line as in [2] (see also $[19,20,33])$. Let $\varepsilon>0$. From [5], there exists $\delta>0$ such that

$$
\overline{c o} F_{\delta}(T(t) ; D)+B_{\delta} \subseteq F_{\varepsilon}(T(t) ; D), \quad \forall t \in S .
$$

From Corollary 1.1 in [5], there exists a natural number $N$ such that

$$
\left\|\frac{1}{N+1} \sum_{i=0}^{N} T\left(t^{i} s\right) y-T(t)\left(\frac{1}{N+1} \sum_{i=0}^{N} T\left(t^{i} s\right) y\right)\right\| \leq \delta,
$$

for all $t, s \in S$ and $y \in D$. Let $t \in S$. Since $\left\{\mu_{n}\right\}$ is left regular, there exists $n_{0} \in \mathbb{N}$ such that

$$
\left\|\mu_{n}-l_{t^{i}}^{*} \mu_{n}\right\| \leq \frac{\delta}{3(K+\|w\|)}
$$

for all $n \geq n_{0}$ and $i=1,2, \ldots, N$. So we have for all $n \geq n_{0}$

$$
\begin{aligned}
& \sup _{y \in D}\left\|T\left(\mu_{n}\right) y-\int \frac{1}{N+1} \sum_{i=0}^{N} T\left(t^{i} s\right) y d \mu_{n}(s)\right\| \\
& \quad=\sup _{y \in D\|z\|=1}\left|\left(\mu_{n}\right)_{s}\langle T(s) y, z\rangle-\left(\mu_{n}\right)_{s}\left\langle\frac{1}{N+1} \sum_{i=0}^{N} T\left(t^{i} s\right) y, z\right\rangle\right| \\
& \quad \leq \frac{1}{N+1} \sum_{i=0}^{N} \sup _{y \in D \| z \sup _{\|}\left|\left(\mu_{n}\right)_{s}\langle T(s) y, z\rangle-\left(l_{t^{i}}^{*} \mu_{n}\right)_{s}\langle T(s) y, z\rangle\right|} \\
& \quad \leq \max _{i=1,2, \ldots, N}\left\|\mu_{n}-l_{t^{i}}^{*} \mu_{n}\right\|(K+\|w\|) \leq \frac{\delta}{3} .
\end{aligned}
$$

Observe, by Lemma 2.2

$$
\int \frac{1}{N+1} \sum_{i=0}^{N} T\left(t^{i} s\right) y d \mu_{n}(s) \in \overline{c o}\left\{\frac{1}{N+1} \sum_{i=0}^{N} T(t)^{i}(T(s) y): s \in S\right\} .
$$

Combining (3.10)-(3.12), we derive

$$
\begin{aligned}
T\left(\mu_{n}\right) y & =\int \frac{1}{N+1} \sum_{i=0}^{N} T\left(t^{i} s\right) y d \mu_{n}(s)+\left(T\left(\mu_{n}\right) y-\int \frac{1}{N+1} \sum_{i=0}^{N} T\left(t^{i} s\right) y d \mu_{n}(s)\right) \\
& \in \overline{c o}\left\{\frac{1}{N+1} \sum_{i=0}^{N} T(t)^{i}(T(s) y): s \in S\right\}+B_{\delta / 3} \\
& \subseteq \overline{c o} F_{\delta}(T(t) ; D)+B_{\delta / 3},
\end{aligned}
$$


for all $y \in D$ and $n \geq n_{0}$. Let $t \in S$ and $\varepsilon>0$. Then there exists $\delta>0$ which satisfies (3.9). Observe

$$
x_{n+1}=T\left(\mu_{n}\right) y_{n}+\frac{\beta}{1-\beta}\left(x_{n+1}-x_{n}\right)+\frac{\alpha_{n}}{1-\beta}\left(\gamma f\left(x_{n}\right)-A T\left(\mu_{n}\right) y_{n}\right) .
$$

Since $\left\|x_{n+1}-x_{n}\right\| \rightarrow 0$ and $\alpha_{n} \rightarrow 0$, there exists $k \in \mathbb{N}$ such that

$$
\begin{aligned}
x_{n+1} & =T\left(\mu_{n}\right) y_{n}+\frac{\beta}{1-\beta}\left(x_{n}-x_{n+1}\right)+\frac{\alpha_{n}}{1-\beta}\left(\gamma f\left(x_{n}\right)-A T\left(\mu_{n}\right) y_{n}\right) \\
& \in \overline{c o} F_{\delta}(T(t) ; D)+B_{\delta / 3}+B_{\delta / 3}+B_{\delta / 3} \\
& \subseteq \overline{c o} F_{\delta}(T(t) ; D)+B_{\delta} \subseteq F_{\varepsilon}(T(t) ; D),
\end{aligned}
$$

for all $n>k$. Hence, $\lim \sup _{n \rightarrow \infty}\left\|x_{n}-T(t) x_{n}\right\| \leq \varepsilon$. Since $\varepsilon>0$ is arbitrary,

$$
\lim _{n \rightarrow \infty}\left\|x_{n}-T(t) x_{n}\right\|=0
$$

We next show that

$$
\lim _{n \rightarrow \infty}\left\|y_{n}-z_{n}\right\|=0
$$

Since $J_{\lambda_{n}}^{M}$ is firmly nonexpansive and $y_{n}=J_{\lambda_{n}}^{M} z_{n}$,

$$
\begin{aligned}
\left\|y_{n}-w\right\|^{2} & =\left\|J_{\lambda_{n}}^{M} z_{n}-J_{\lambda_{n}}^{M} w\right\|^{2} \\
& \leq\left\langle J_{\lambda_{n}}^{M} z_{n}-J_{\lambda_{n}}^{M} w, z_{n}-w\right\rangle \\
& =\left\langle y_{n}-w, z_{n}-w\right\rangle \\
& =\frac{1}{2}\left(\left\|y_{n}-w\right\|^{2}+\left\|z_{n}-w\right\|^{2}-\left\|z_{n}-y_{n}\right\|^{2}\right),
\end{aligned}
$$

which implies

$$
\left\|y_{n}-w\right\|^{2} \leq\left\|z_{n}-w\right\|^{2}-\left\|z_{n}-y_{n}\right\|^{2} .
$$

Therefore,

$$
\begin{aligned}
\left\|x_{n+1}-w\right\|^{2}= & \left\|\left[(1-\beta)\left(T\left(\mu_{n}\right) y_{n}-w\right)+\beta\left(x_{n}-w\right)\right]+\alpha_{n}\left[\gamma f\left(x_{n}\right)-A T\left(\mu_{n}\right) y_{n}\right]\right\|^{2} \\
\leq & \left\|(1-\beta)\left(T\left(\mu_{n}\right) y_{n}-w\right)+\beta\left(x_{n}-w\right)\right\|^{2} \\
& +2 \alpha_{n}\left\langle\gamma f\left(x_{n}\right)-A T\left(\mu_{n}\right) y_{n}, x_{n+1}-w\right\rangle \\
\leq & (1-\beta)\left\|y_{n}-w\right\|^{2}+\beta\left\|x_{n}-w\right\|^{2} \\
& +2 \alpha_{n}\left\langle\gamma f\left(x_{n}\right)-A T\left(\mu_{n}\right) y_{n}, x_{n+1}-w\right\rangle \\
\leq & (1-\beta)\left(\left\|z_{n}-w\right\|^{2}-\left\|z_{n}-y_{n}\right\|^{2}\right)+\beta\left\|x_{n}-w\right\|^{2} \\
& +2 \alpha_{n}\left\langle\gamma f\left(x_{n}\right)-A T\left(\mu_{n}\right) y_{n}, x_{n+1}-w\right\rangle \\
\leq & \left\|x_{n}-w\right\|^{2}-(1-\beta)\left\|z_{n}-y_{n}\right\|^{2} \\
& +2 \alpha_{n}\left\langle\gamma f\left(x_{n}\right)-A T\left(\mu_{n}\right) y_{n}, x_{n+1}-w\right\rangle
\end{aligned}
$$


which yields

$$
(1-\beta)\left\|z_{n}-y_{n}\right\|^{2} \leq \alpha_{n} M_{2}+\left(\left\|x_{n}-w\right\|^{2}-\left\|x_{n+1}-w\right\|^{2}\right)
$$

for some $M_{2}>0$. Thus, (3.15) holds by (3.8) and $\alpha_{n} \rightarrow 0$.

We next show that

$$
\lim _{n \rightarrow \infty}\left\|x_{n}-T x_{n}\right\|=0 .
$$

From (3.2), we have

$$
\left\|y_{n}-w\right\|^{2} \leq\left\|z_{n}-w\right\|^{2} \leq\left\|x_{n}-w\right\|^{2}+\left(1-\delta_{n}\right)\left(\kappa-\delta_{n}\right)\left\|x_{n}-T x_{n}\right\|^{2} .
$$

So, we obtain

$$
\begin{aligned}
\left\|x_{n+1}-w\right\|^{2} \leq & (1-\beta)\left\|y_{n}-w\right\|^{2}+\beta\left\|x_{n}-w\right\|^{2} \\
& +2 \alpha_{n}\left\langle\gamma f\left(x_{n}\right)-A T\left(\mu_{n}\right) y_{n}, x_{n+1}-w\right\rangle \\
\leq & (1-\beta)\left(\left\|x_{n}-w\right\|^{2}+\left(1-\delta_{n}\right)\left(\kappa-\delta_{n}\right)\left\|x_{n}-T x_{n}\right\|^{2}\right)+\beta\left\|x_{n}-w\right\|^{2} \\
& +2 \alpha_{n}\left(\gamma f\left(x_{n}\right)-A T\left(\mu_{n}\right) y_{n}, x_{n+1}-w\right\rangle \\
\leq & \left\|x_{n}-w\right\|^{2}+(1-\beta)\left(1-\delta_{n}\right)\left(\kappa-\delta_{n}\right)\left\|x_{n}-T x_{n}\right\|^{2}+\alpha_{n} M_{2} .
\end{aligned}
$$

It follows that

$$
(1-\beta)\left(1-\delta_{n}\right)\left(\delta_{n}-\kappa\right)\left\|x_{n}-T x_{n}\right\|^{2} \leq \alpha_{n} M_{2}+\left\|x_{n}-w\right\|^{2}-\left\|x_{n+1}-w\right\|^{2} .
$$

From (C1) and (C3), we conclude that (3.16) holds. Moreover, we get that

$$
\lim _{n \rightarrow \infty}\left\|x_{n}-z_{n}\right\|=0
$$

It is easy to see that $P_{F}(\gamma f+(I-A))$ is a contraction. So, by Banach's contraction principle, there exists a unique point $p$ which satisfies the following variational inequality:

$$
\langle(\gamma f-A) p, q-p\rangle \leq 0, \quad \forall q \in F
$$

We next show that

$$
\limsup _{n \rightarrow \infty}\left\langle(\gamma f-A) p, x_{n}-p\right\rangle \leq 0
$$

To this end, we choose a subsequence $\left\{x_{n_{k}}\right\}$ of $\left\{x_{n}\right\}$ such that

$$
\limsup _{n \rightarrow \infty}\left\langle(\gamma f-A) p, x_{n}-p\right\rangle=\lim _{k \rightarrow \infty}\left\langle(\gamma f-A) p, x_{n_{k}}-p\right\rangle .
$$

Since $\left\{x_{n}\right\}$ is bounded and $H$ is reflexive, there exists a point $z \in H$ such that $x_{n_{k}} \rightarrow z$. From (3.15) and (3.17), there exists a corresponding subsequence $\left\{y_{n_{k}}\right\}$ of $\left\{y_{n}\right\}$ (resp. $\left\{z_{n_{k}}\right\}$ of $\left\{z_{n}\right\}$ ) such that $y_{n_{k}} \rightarrow z$ (resp. $\left.z_{n_{k}} \rightarrow z\right)$. 
We next show that $z \in M^{-1}(0)$. Since $y_{n}=J_{\lambda_{n}}^{M} z_{n}$,

$$
\left\|A_{\lambda_{n}} z_{n}\right\|=\frac{1}{\lambda_{n}}\left\|y_{n}-z_{n}\right\|
$$

From (3.15) and $\liminf _{n \rightarrow \infty} \lambda_{n}>0$, we have

$$
\lim _{n \rightarrow \infty}\left\|A_{\lambda_{n}} z_{n}\right\|=0
$$

Noting that $\left(z_{n}, A_{\lambda_{n}} z_{n}\right) \in G(M)$, by the monotonicity of $M$, we have

$$
\left\langle s-z_{n}, s^{*}-A_{\lambda_{n}} z_{n}\right\rangle \geq 0
$$

for all $\left(s, s^{*}\right) \in G(M)$. So we obtain

$$
\left\langle s-z, s^{*}\right\rangle \geq 0
$$

for all $\left(s, s^{*}\right) \in G(M)$. Hence, $z \in M^{-1}(0)$ by the maximality of $M$.

On the other hand, from (3.14), we get that $z \in F(\mathcal{S})$ by the demiclosedness of a nonexpansive mapping $[4,12]$. Applying Lemma 2.7 to (3.16), we also get that $z \in F(T)$. This shows that $z \in F$, and hence

$$
\limsup _{n \rightarrow \infty}\left\langle(\gamma f-A) p, x_{n}-p\right\rangle=\langle(\gamma f-A) p, z-p\rangle \leq 0
$$

We finally show that $x_{n} \rightarrow p$ as $n \rightarrow \infty$. From Lemmas 2.3 and 2.4 , we have

$$
\begin{aligned}
\left\|x_{n+1}-p\right\|^{2}= & \left\|\left[\left((1-\beta) I-\alpha_{n} A\right)\left(T\left(\mu_{n}\right) y_{n}-p\right)+\beta\left(x_{n}-p\right)\right]+\alpha_{n}\left(\gamma f\left(x_{n}\right)-A p\right)\right\|^{2} \\
\leq & \left\|\left((1-\beta) I-\alpha_{n} A\right)\left(T\left(\mu_{n}\right) y_{n}-p\right)+\beta\left(x_{n}-p\right)\right\|^{2} \\
& +2 \alpha_{n}\left\langle\gamma f\left(x_{n}\right)-A p, x_{n+1}-p\right\rangle \\
= & \left\|(1-\beta) \frac{(1-\beta) I-\alpha_{n} A}{(1-\beta)}\left(T\left(\mu_{n}\right) y_{n}-p\right)+\beta\left(x_{n}-p\right)\right\|^{2} \\
& +2 \alpha_{n} \gamma\left\langle f\left(x_{n}\right)-f(p), x_{n+1}-p\right\rangle+2 \alpha_{n}\left\langle\gamma f(p)-A p, x_{n+1}-p\right\rangle \\
\leq & (1-\beta)\left\|\frac{(1-\beta) I-\alpha_{n} A}{(1-\beta)}\left(T\left(\mu_{n}\right) y_{n}-p\right)\right\|^{2}+\beta\left\|x_{n}-p\right\|^{2} \\
& +2 \alpha_{n} \gamma \alpha\left\|x_{n}-p\right\|\left\|x_{n+1}-p\right\|+2 \alpha_{n}\left\langle\gamma f(p)-A p, x_{n+1}-p\right\rangle \\
\leq & \frac{(1-\beta) I-\alpha_{n} A \|^{2}}{1-\beta}\left\|T\left(\mu_{n}\right) y_{n}-p\right\|^{2}+\beta\left\|x_{n}-p\right\|^{2} \\
& +\alpha_{n} \gamma \alpha\left(\left\|x_{n}-p\right\|^{2}+\left\|x_{n+1}-p\right\|^{2}\right)+2 \alpha_{n}\left\langle\gamma f(p)-A p, x_{n+1}-p\right\rangle \\
\leq & \left(\frac{\left((1-\beta)-\bar{\gamma} \alpha_{n}\right)^{2}}{1-\beta}+\beta+\alpha_{n} \gamma \alpha\right)\left\|x_{n}-p\right\|^{2}+\alpha_{n} \gamma \alpha\left\|x_{n+1}-p\right\|^{2} \\
& +2 \alpha_{n}\left\langle\gamma f(p)-A p, x_{n+1}-p\right\rangle \\
= & \left(1-(2 \bar{\gamma}-\alpha \gamma) \alpha_{n}+\frac{\bar{\gamma}^{2} \alpha_{n}^{2}}{1-\beta}\right)\left\|x_{n}-p\right\|^{2}+\alpha_{n} \gamma \alpha\left\|x_{n+1}-p\right\|^{2} \\
& +2 \alpha_{n}\left\langle\gamma f(p)-A p, x_{n+1}-p\right\rangle .
\end{aligned}
$$


It follows that

$$
\begin{aligned}
\left\|x_{n+1}-p\right\|^{2} \leq & \left(1-\frac{2 \alpha_{n}(\bar{\gamma}-\alpha \gamma)}{1-\alpha \gamma \alpha_{n}}\right)\left\|x_{n}-p\right\|^{2} \\
& +\frac{2 \alpha_{n}(\bar{\gamma}-\alpha \gamma)}{1-\alpha \gamma \alpha_{n}}\left(\frac{1}{\bar{\gamma}-\alpha \gamma}\left\langle\gamma f(p)-A p, x_{n+1}-p\right\rangle\right. \\
& \left.+\frac{\bar{\gamma}^{2} \alpha_{n}}{2(1-\beta)(\bar{\gamma}-\alpha \gamma)}\left\|x_{n}-p\right\|^{2}\right) .
\end{aligned}
$$

From (3.19) and (C1), we can apply Lemma 2.5 to conclude that $x_{n} \rightarrow p$ as $n \rightarrow \infty$. This completes the proof.

From Rockafellar's theorem [29, 30], we next apply our result to the convex minimization problem in a Hilbert space.

Corollary 3.2 Let $H$ be a real Hilbert space and $\mathcal{S}=\{T(t): t \in S\}$ a nonexpansive semigroup on $H$. Let $g: H \rightarrow(-\infty, \infty]$ be a proper lower semi-continuous convex function and $T: H \rightarrow H$ a $\kappa$-strict pseudocontraction such that $F:=F(\mathcal{S}) \cap \partial g^{-1}(0) \cap F(T) \neq \emptyset$. Let $X$ be a left invariant subspace of $\ell^{\infty}(S)$ such that $1 \in X$, and the function $t \mapsto\langle T(t) x, y\rangle$ is an element of $X$ for each $x, y \in H$. Let $\left\{\mu_{n}\right\}$ be a left regular sequence of means on $X$ such that $\lim _{n \rightarrow \infty}\left\|\mu_{n+1}-\mu_{n}\right\|=0$. Let $f$ be an $\alpha$-contraction on $H$ and $A$ a strongly positive bounded linear operator with coefficient $\bar{\gamma}$. Let $\left\{\alpha_{n}\right\}, \beta, \gamma,\left\{\delta_{n}\right\}$ and $\left\{\lambda_{n}\right\}$ be as in Theorem 3.1. Then the sequence $\left\{x_{n}\right\}$ generated by $x_{1} \in H$ and

$$
\left\{\begin{array}{l}
z_{n}=\delta_{n} x_{n}+\left(1-\delta_{n}\right) T x_{n}, \\
y_{n}=\operatorname{argmin}_{y \in H}\left\{g(y)+\frac{1}{2 \lambda_{n}}\left\|z_{n}-y\right\|^{2}\right\}, \\
x_{n+1}=\alpha_{n} \gamma f\left(x_{n}\right)+\beta x_{n}+\left((1-\beta) I-\alpha_{n} A\right) T\left(\mu_{n}\right) y_{n}, \quad n \geq 1,
\end{array}\right.
$$

converges strongly to $p \in F$ which also solves the variational inequality (3.1).

Using Lemma 2.8, we next apply our result to a finite family of strict pseudocontractions in a Hilbert space.

Corollary 3.3 Let $H$ be a real Hilbert space and $\mathcal{S}=\{T(t): t \in S\}$ a nonexpansive semigroup on $H$. Let $M: H \rightarrow 2^{H}$ be a maximal monotone operator and $\left\{T_{i}\right\}_{i=1}^{N}: H \rightarrow H$ a family of $\kappa_{i}$-strict pseudocontractions such that $F:=F(\mathcal{S}) \cap M^{-1}(0) \cap F\left(T_{1}\right) \cap \cdots \cap F\left(T_{N}\right) \neq \emptyset$. Let $\kappa=\max \left\{\kappa_{i}: 1 \leq i \leq N\right\}$. Let $X$ be a left invariant subspace of $\ell^{\infty}(S)$ such that $1 \in X$, and the function $t \mapsto\langle T(t) x, y\rangle$ is an element of $X$ for each $x, y \in H$. Let $\left\{\mu_{n}\right\}$ be a left regular sequence of means on $X$ such that $\lim _{n \rightarrow \infty}\left\|\mu_{n+1}-\mu_{n}\right\|=0$. Let $f$ be an $\alpha$-contraction on $H$ and $A$ a strongly positive bounded linear operator with coefficient $\bar{\gamma}$. Let $\left\{\alpha_{n}\right\}, \beta, \gamma,\left\{\delta_{n}\right\}$ and $\left\{\lambda_{n}\right\}$ be as in Theorem 3.1 and $\eta_{i} \in(0,1)$ with $\sum_{i=1}^{N} \eta_{i}=1$. Then the sequence $\left\{x_{n}\right\}$ generated by $x_{1} \in H$ and

$$
\left\{\begin{array}{l}
y_{n}=J_{\lambda_{n}}^{M}\left(\delta_{n} x_{n}+\left(1-\delta_{n}\right) \sum_{i=1}^{N} \eta_{i} T_{i} x_{n}\right), \\
x_{n+1}=\alpha_{n} \gamma f\left(x_{n}\right)+\beta x_{n}+\left((1-\beta) I-\alpha_{n} A\right) T\left(\mu_{n}\right) y_{n}, \quad n \geq 1,
\end{array}\right.
$$

converges strongly to $p \in F$ which also solves the variational inequality (3.1). 
Using the results proved in [37] (see also [19]), we obtain the following corollaries.

Corollary 3.4 Let $H$ be a real Hilbert space. Let $S_{1}$ and $S_{2}$ be nonexpansive mappings on $H$ with $S_{1} S_{2}=S_{2} S_{1}$. Let $M: H \rightarrow 2^{H}$ be a maximal monotone operator and let $T: H \rightarrow H$ be a $\kappa$-strict pseudocontraction such that $F:=F\left(S_{1}\right) \cap F\left(S_{2}\right) \cap M^{-1}(0) \cap F(T) \neq \emptyset$. Let $f$ be an $\alpha$-contraction on $H$ and $A$ a strongly positive bounded linear operator with coefficient $\bar{\gamma}$. Let $\left\{\alpha_{n}\right\}, \beta, \gamma,\left\{\delta_{n}\right\}$, and $\left\{\lambda_{n}\right\}$ be as in Theorem 3.1. Then the sequence $\left\{x_{n}\right\}$ generated by $x_{1} \in H$ and

$$
\left\{\begin{array}{l}
y_{n}=J_{\lambda_{n}}^{M}\left(\delta_{n} x_{n}+\left(1-\delta_{n}\right) T x_{n}\right), \\
x_{n+1}=\alpha_{n} \gamma f\left(x_{n}\right)+\beta x_{n}+\left((1-\beta) I-\alpha_{n} A\right)\left(\frac{1}{n^{2}} \sum_{i=0}^{n-1} \sum_{j=0}^{n-1} S_{1}^{i} S_{2}^{j} y_{n}\right), \quad n \geq 1,
\end{array}\right.
$$

converges strongly to $p \in F$ which also solves the variational inequality (3.1).

Corollary 3.5 Let H be a real Hilbert space. Let $\mathcal{S}=\left\{T(t): t \in \mathbb{R}_{+}\right\}$be a strongly continuous nonexpansive semigroup on $H$. Let $M: H \rightarrow 2^{H}$ be a maximal monotone operator and $T: H \rightarrow H$ a $\kappa$-strict pseudocontraction such that $F:=F(\mathcal{S}) \cap M^{-1}(0) \cap F(T) \neq \emptyset$. Let $f$ be an $\alpha$-contraction on $H$ and $A$ a strongly positive bounded linear operator with coefficient $\bar{\gamma}$. Let $\left\{\alpha_{n}\right\}, \beta, \gamma,\left\{\delta_{n}\right\}$, and $\left\{\lambda_{n}\right\}$ be as in Theorem 3.1. Then the sequence $\left\{x_{n}\right\}$ generated by $x_{1} \in H$ and

$$
\left\{\begin{array}{l}
y_{n}=J_{\lambda_{n}}^{M}\left(\delta_{n} x_{n}+\left(1-\delta_{n}\right) T x_{n}\right), \\
x_{n+1}=\alpha_{n} \gamma f\left(x_{n}\right)+\beta x_{n}+\left((1-\beta) I-\alpha_{n} A\right)\left(\frac{1}{t_{n}} \int_{0}^{t_{n}} T(s) y_{n} d(s)\right), \quad n \geq 1,
\end{array}\right.
$$

where $\left\{t_{n}\right\}$ is an increasing sequence in $(0, \infty)$ such that $\lim _{n \rightarrow \infty} t_{n}=\infty$ and $\lim _{n \rightarrow \infty} t_{n}$ l $t_{n+1}=1$, converges strongly to $p \in F$ which also solves the variational inequality (3.1).

Corollary 3.6 Let $H$ be a real Hilbert space. Let $\mathcal{S}=\left\{T(t): t \in \mathbb{R}_{+}\right\}$be a strongly continuous nonexpansive semigroup on $H$. Let $M: H \rightarrow 2^{H}$ be a maximal monotone operator and $T: H \rightarrow H$ a $\kappa$-strict pseudocontraction such that $F:=F(\mathcal{S}) \cap M^{-1}(0) \cap F(T) \neq \emptyset$. Let $f$ be an $\alpha$-contraction on $H$ and $A$ a strongly positive bounded linear operator with coefficient $\bar{\gamma}$. Let $\left\{\alpha_{n}\right\}, \beta, \gamma,\left\{\delta_{n}\right\}$ and $\left\{\lambda_{n}\right\}$ be as in Theorem 3.1. Then the sequence $\left\{x_{n}\right\}$ generated by $x_{1} \in H$ and

$$
\left\{\begin{array}{l}
y_{n}=J_{\lambda_{n}}^{M}\left(\delta_{n} x_{n}+\left(1-\delta_{n}\right) T x_{n}\right) \\
x_{n+1}=\alpha_{n} \gamma f\left(x_{n}\right)+\beta x_{n}+\left((1-\beta) I-\alpha_{n} A\right)\left(a_{n} \int_{0}^{\infty} \exp \left(-a_{n} s\right) T(s) y_{n} d(s)\right), \quad n \geq 1
\end{array}\right.
$$

where $\left\{a_{n}\right\}$ is a decreasing sequence in $(0, \infty)$ such that $\lim _{n \rightarrow \infty} a_{n}=0$, converges strongly to $p \in F$ which also solves the variational inequality (3.1).

Competing interests

The authors declare that they have no competing interests.

\section{Acknowledgement}

The author wishes to thank Professor Anthony To-Ming Lau for the hospitality and guidance when stayed in University of Alberta during Spring/Summer 2011 and Professor Suthep Suantai for the valuable suggestion. The author was supported by the Thailand Research Fund, the Commission on Higher Education, and University of Phayao under Grant MRG5580016. 


\section{References}

1. Acedo, GL, Xu, HK: Iterative methods for strict pseudo-contractions in Hilbert spaces. Nonlinear Anal. TMA 67 2258-2271 (2007)

2. Atsushiba, S, Takahashi, W: Approximation common fixed points of nonexpansive semigroups by the Mann iteration process. Ann. Univ. Mariae Curie-SkT̄odowska, Sect. A 51, 1-16 (1997)

3. Browder, FE, Petryshyn, WV: Construction of fixed points of nonlinear mappings in Hilbert spaces. J. Math. Anal. Appl. 20, 197-228 (1967)

4. Browder, FE: Nonexpansive nonlinear operators in a Banach space. Proc. Natl. Acad. Sci. USA 54, 1041-1044 (1965)

5. Bruck, RE: On the convex approximation property and the asymptotic behavior of nonlinear contractions in Banach spaces. Isr. J. Math. 38, 304-314 (1981)

6. Chen, $\mathrm{R}, \mathrm{He}, \mathrm{H}$ : Viscosity approximation of common fixed points of nonexpansive semigroups in Banach space. Appl. Math. Lett. 20,751-757 (2007)

7. Chen, R, Song, Y: Convergence to common fixed point of nonexpansive semigroups. J. Comput. Appl. Math. 200 566-575 (2007)

8. Cho, YJ, Kang, SM, Zhou, H: Approximate proximal point algorithms for finding zeroes of maximal monotone operators in Hilbert spaces. J. Inequal. Appl. 2008, Art. ID 598191 (2008)

9. Cholamjiak, P, Suantai, S: Viscosity approximation methods for a nonexpansive semigroup in Banach spaces with gauge functions. J. Glob. Optim. (2011). doi:10.1007/s10898-011-9756-4

10. Cholamjiak, P, Cho, YJ, Suantai, S: Composite iterative schemes for maximal monotone operators in reflexive Banach spaces. Fixed Point Theory Appl. 2011, 7 (2011)

11. Day, MM: Amenable semigroup. III. J. Math. 1, 509-544 (1957)

12. Goebel, K, Kirk, WA: Topics in Metric Fixed Point Theory. Cambridge Studies in Advanced Mathematics, vol. 28. Cambridge University Press, Cambridge, UK (1990)

13. Halpern, B. Fixed points of nonexpanding maps. Bull. Am. Math. Soc. 73, 957-961 (1967)

14. Kamimura, S, Takahashi, W: Approximating solutions of maximal monotone operators in Hilbert spaces. J. Approx. Theory 106, 226-240 (2000)

15. Kohsaka, F, Takahashi, W: Proximal point algorithms with Bregman functions in Banach spaces. J. Nonlinear Convex Anal. 6, 505-523 (2005)

16. Lau, AT-M: Invariant means on almost periodic functions and fixed point properties. Rocky Mt. J. Math. 3, 69-76 (1973)

17. Lau, AT-M: Invariant means and fixed point properties of semigroup of nonexpansive mappings. Taiwan. J. Math. 12, 1525-1542 (2008)

18. Lau, AT-M, Takahashi, W: Invariant means and fixed point properties for nonexpansive representations of topological semigroups. Topol. Methods Nonlinear Anal. 5, 39-57 (1995)

19. Lau, AT-M, Miyake, H, Takahashi, W: Approximation of fixed points for amenable semigroups of nonexpansive mappings in Banach spaces. Nonlinear Anal. TMA 67, 1211-1225 (2007)

20. Lau, AT-M, Shioji, N, Takahashi, W: Existence of nonexpansive retractions for amenable semigroups of nonexpansive mappings and nonlinear ergodic theorems in Banach spaces. J. Funct. Anal. 161, 62-75 (1999)

21. Mann, WR: Mean value methods in iterations. Proc. Am. Math. Soc. 4, 506-510 (1953)

22. Marino, G, Xu, HK: A general iterative method for nonexpansive mappings in Hilbert spaces. J. Math. Anal. Appl. 318 43-52 (2006)

23. Marino, G, Xu, HK: Weak and strong convergence theorems for strict pseudo-contractions in Hilbert spaces. J. Math. Anal. Appl. 329, 336-346 (2007)

24. Martinet, B: Régularisation d'inéquations variationelles par approximations successives. Rev. Francaise d'Informatique et de Recherche Opérationelle 4, 154-159 (1970)

25. Moudafi, A: Viscosity approximation methods for fixed point problems. J. Math. Anal. Appl. 241, 46-55 (2000)

26. Petruşel, A, Yao, J-C: Viscosity approximation to common fixed points of families of nonexpansive mappings with generalized contractions mappings. Nonlinear Anal. TMA 69, 1100-1111 (2008)

27. Qin, X, Kang, SM, Cho, YJ: Approximating zeros of monotone operators by proximal point algorithms. J. Glob. Optim. 46, 75-87 (2010)

28. Qin, X, Shang, M, Kang, SM: Strong convergence theorems of modified Mann iterative process for strict pseudo-contractions in Hilbert spaces. Nonlinear Anal. TMA 70, 1257-1264 (2009)

29. Rockafellar, RT: On the maximality of suns of nonlinear monotone operators. Trans. Am. Math. Soc. 149, 75-88 (1970)

30. Rockafellar, RT: Monotone operators and the proximal point algorithm. SIAM J. Control Optim. 14, $877-898$ (1976)

31. Saeidi, S: Existence of ergodic retractions for semigroups in Banach spaces. Nonlinear Anal. TMA 69, 3417-3422 (2008)

32. Saeidi, S: Iterative algorithms for finding common solutions of variational inequalities and systems of equilibrium problems and fixed points of families and semigroups of nonexpansive mappings. Nonlinear Anal. TMA 70, 4195-4208 (2009)

33. Shioji, N, Takahashi, W: Strong convergence of average approximants for asymptotically nonexpansive mappings in Banach spaces. J. Approx. Theory 97, 53-64 (1999)

34. Solodov, MV, Svaiter, BF: Forcing strong convergence of proximal point iterations in a Hilbert space. Math. Program. 87, 189-202 (2000)

35. Song, Y, Xu, S: Strong convergence theorems for nonexpansive semigroup in Banach spaces. J. Math. Anal. Appl. 338 152-161 (2008)

36. Suzuki, T: Strong convergence of Krasnoselskii and Mann's type sequences for one parameter nonexpansive semigroups without Bochner integrals. J. Math. Anal. Appl. 305, 227-239 (2005)

37. Takahashi, W: Nonlinear Function Analysis. Yokohama Publishers, Yokohama (2000)

38. Takahashi, W: Viscosity approximation methods for countable families of nonexpansive mappings in Banach spaces. Nonlinear Anal. TMA 70, 719-734 (2009)

39. Takahashi, W: A nonlinear ergodic theorem for an amenable semigroup of nonexpansive mappings in a Hilbert space. Proc. Am. Math. Soc. 81, 253-256 (1981)

40. Wang, S, Wang, F: On relaxed and contraction-proximal point algorithms in Hilbert spaces. J. Inequal. Appl. 2011, 41 (2011) 
41. Xu, HK: Iterative algorithms for nonlinear operators. J. Lond. Math. Soc. 66, 240-256 (2002)

42. Xu, HK: Viscosity approximation methods for nonexpansive mappings. J. Math. Anal. Appl. 298, 279-291 (2004)

43. Xu, HK: A strong convergence theorem for contraction semigroups in Banach spaces. Bull. Aust. Math. Soc. 72 , 371-379 (2005)

44. Zhou, $\mathrm{H}$ : Convergence theorems of fixed points for Lipschitz pseudo-contractions in Hilbert spaces. J. Math. Anal. Appl. 343, 546-556 (2008)

doi:10.1186/1687-1812-2012-129

Cite this article as: Cholamjiak: Convergence results for the zero-finding problem and fixed points of nonexpansive semigroups and strict pseudocontractions. Fixed Point Theory and Applications 2012 2012:129.

Submit your manuscript to a SpringerOpen ${ }^{\circ}$ journal and benefit from:

- Convenient online submission

Rigorous peer review

- Immediate publication on acceptance

Open access: articles freely available online

- High visibility within the field

- Retaining the copyright to your article 